\section{Application of Marker-assisted Selection in Persimmon Breeding of PCNA Offspring Using SCAR Markers among the Population from the Cross between Non-PCNA 'Taigetsu' and PCNA 'Kanshu'}

Nobuhito Mitani ${ }^{1,6}$, Atsushi Kono, Masahiko Yamada ${ }^{2}$, Akihiko Sato, Shozo Kobayashi, Yusuke Ban, Toshihito Ueno ${ }^{3}$, and Mikio Shiraishi ${ }^{4}$ Grape and Persimmon Research Division, National Agriculture and Food Research Organization (NARO) Institute of Fruit Tree Science (NIFTS), Akitsu, Higashihiroshima, Hiroshima, 739-2494, Japan

\author{
Shinya Kanzaki \\ Laboratory of Horticultural Science, Faculty of Agriculture, Kinki \\ University, Nara, 631-8505, Japan
}

Tomoyuki Tsujimoto 5 and Keizo Yonemori

Laboratory of Pomology, Graduate School of Agriculture, Kyoto University, Sakyo-ku, Kyoto, 606-8502, Japan

Additional index words. astringency, Diospyros kaki, inheritance, polyploidy

\begin{abstract}
Persimmon (Diospyros kaki Thunb) is hexaploid, and the pollination-constant, non-astringent (PCNA)/non-PCNA trait of Japanese origin is qualitatively controlled by the AST/ast alleles at a single locus and the PCNA trait is recessive to the non-PCNA trait. To avoid inbreeding depression led by repeated crosses among PCNA genotypes, non-PCNA genotypes should be used as cross parents. The marker-assisted selection system has been developed for the selection of PCNA offspring in the progeny derived from the cross of nonPCNA 'Taigetsu' (non-PCNA 'Kurokuma' $\times$ PCNA 'Taishu') to PCNA 'Kanshu'. The primer pairs E8.5/E9r and 7H9F/AST-R were used for detecting the molecular markers $A_{1}$ and $\mathrm{A}_{3}$, respectively, which link $A S T$ alleles. Complete agreement was found between the sequence-characterized amplified region (SCAR) marker genotype and fruit astringency phenotype of the 48 offspring. The result confirmed that the marker-assisted selection using those markers was highly practical. In a larger offspring population (522 offspring) from the same cross, offspring segregated into 100 with both markers, 162 with only $A_{1}, 179$ with $A_{3}$, and 81 with neither, and this segregation ratio was significantly different from 2:3:3:2, which is the segregation ratio of random chromosome assortment in autohexaploid. The percentage of offspring expected to be PCNA was $15.5 \%$ (81 of 522), which was slightly lower than $20 \%$.
\end{abstract}

Received for publication 1 Apr. 2014. Accepted for publication 22 June 2014

We thank T. Nakasumi, F. Umeda, and Y. Okuyama of NIFTS for their cooperation and assistance with sampling and polymerase chain reaction analysis; $\mathrm{T}$. Akagi of Kyoto University for his helpful discussion; and A. Katayama-Ikegami of Ishikawa Prefectural University and N. Onoue of NIFTS for their critical reading of the manuscript.

${ }^{1}$ Present address: Plant Physiology and Fruit Chemistry Division, NIFTS, Tsukuba, Ibaraki 305-8605, Japan.

${ }^{2}$ Present address: NIFTS, Tsukuba, Ibaraki 305-8605, Japan.

${ }^{3}$ Present address: Yamanashi Fruit Tree Experiment Station, Yamanashi, Yamanashi, 405-0043, Japan.

${ }^{4}$ Present address: Fukuoka Agriculture Research Center, Chikushino, Fukuoka, 818-8549, Japan.

${ }^{5}$ Present address: Nara Fruit Tree Research Center, Gojo, Nara, 637-0105, Japan.

${ }^{6}$ To whom reprint requests should be addressed; e-mail nobuhi@affrc.go.jp. trait (Ikeda et al., 1985; Yamada, 2005). Almost no PCNA $F_{1}$ offspring were obtained from crosses between PCNA and non-PCNA local cultivars or from crosses among non-PCNA local cultivars of Japanese origin (Ikeda et al., 1985). When a non-PCNA cultivar, Nishimurawase, was crossed with six PCNA cultivars and selections, no PCNA offspring were yielded among 95 offspring (Yamada and Sato, 2002).

Therefore, in the breeding program of the National Agriculture and Food Research Organization Institute of Fruit Tree Science (NIFTS), crosses have been made primarily among PCNA cultivars/selections to obtain PCNA offspring. However, repeated crosses among PCNA cultivars/selections has led to inbreeding depression for tree vigor, productivity, and fruit weight (Yamada, 1993, 2005). To avoid this inbreeding depression, the backcross approach with non-PCNA cultivars/selections has also been used for PCNA breeding. 'Taigetsu' and 'Taiten' are new non-PCNA cultivars selected from a cross between 'Kurokuma' (non-PCNA) and 'Taishu' (PCNA) (Yamada et al., 2012a, 2012b). The trees of both cultivars are vigorous and highly productive, and their fruits are very large. Accordingly, the use of these cultivars as cross parents could aid in eliminating the inbreeding depression. However, the backcross of non-PCNA $F_{1}$ offspring derived from PCNA $\times$ nonPCNA to PCNA yielded only $14.5 \%$ PCNA offspring on average (Ikeda et al., 1985). So marker-assisted selection should be developed for selecting PCNA offspring efficiently.

Most persimmon cultivars are hexaploid (Tamura et al., 1998), and segregation analysis of molecular markers has indicated hexasomic inheritance with six alleles in the single $A S T$ locus (Akagi et al., 2010; Kanzaki et al., 2008). The presence of one dominant $A S T$ allele is sufficient to express the non-PCNA trait, and the PCNA trait is expressed only when all of the alleles present at the locus are recessive ast alleles (denoted as aaaaaa). Molecular markers linked to $A S T$ alleles are reported and these $A S T$-linked regions have polymorphism. To date, three types of molecular markers linked to AST alleles, $A_{1}, A_{2}$, and $A_{3}$, have been reported (Kanzaki et al., 2008, 2009, 2010). The genotype of 'Kurokuma' is reported to be $\mathrm{A}_{1} \mathrm{~A}_{2} \mathrm{~A}_{3}$ aaa, and those of 'Taigetsu' and 'Taiten' are $\mathrm{A}_{1} \mathrm{~A}_{3}$ aaaa and $\mathrm{A}_{2} \mathrm{~A}_{3}$ aaaa, respectively (Akagi et al., 2012; Kanzaki et al., 2009, 2010). The astringency types of the offspring populations from the cross between 'Taiten' and PCNA have been identified by two SCAR markers (Mitani et al., 2014).

The purpose of the present study is to confirm that SCAR markers could reliably distinguish PCNA and non-PCNA genotypes in a large number of offspring derived from the backcross between 'Taigetsu' and PCNA 'Kanshu'. Furthermore, to reveal the mode of inheritance for the $A S T$ locus, we estimate the 

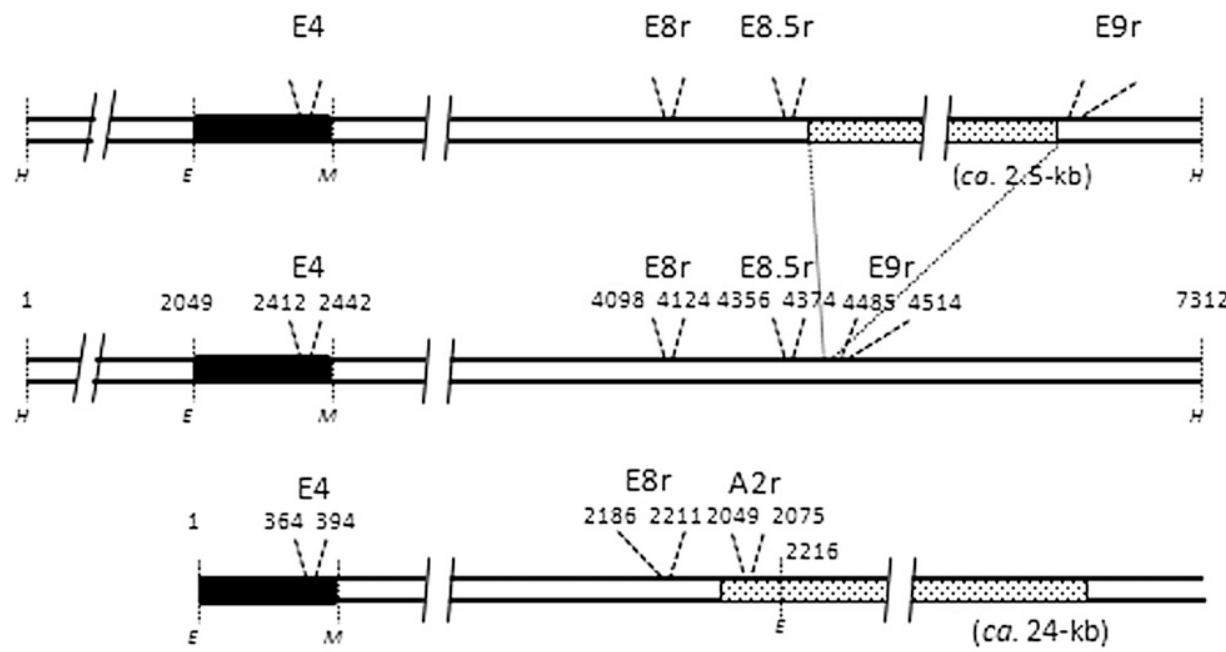

E8r A2r

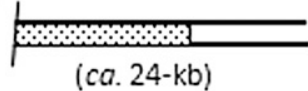

(ca. 24-kb)
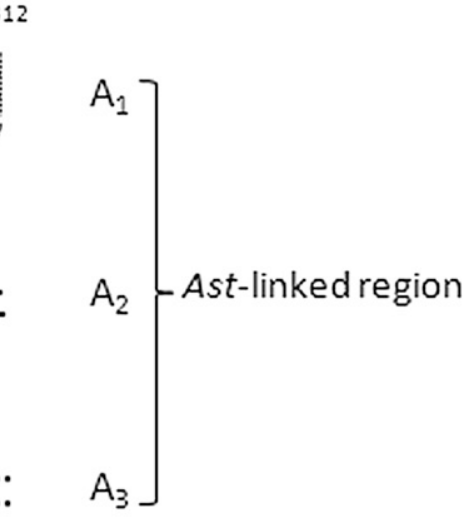

deletion (ca. 11.5-kb)

Fig. 1. Schematic diagram of Ast-linked genomic regions. The black boxes indicate the initially isolated random amplified fragment polymorphism (RFLP) fragment, and the numbers in the $\mathrm{A}_{1}$ and $\mathrm{A}_{2}$ regions show primer positions from the left end of the isolated amplified fragment length polymorphism (AFLP) fragment (Kanzaki et al., 2001, 2009). E, M, and H represent the EcoRI, MseI, and HindIII restriction enzyme sites, respectively. The light gray boxes indicate the retrotransposon-like inserts. The dotted region in $\mathrm{A}_{3}$ indicates the large deletion (Akagi et al., 2010, 2012).

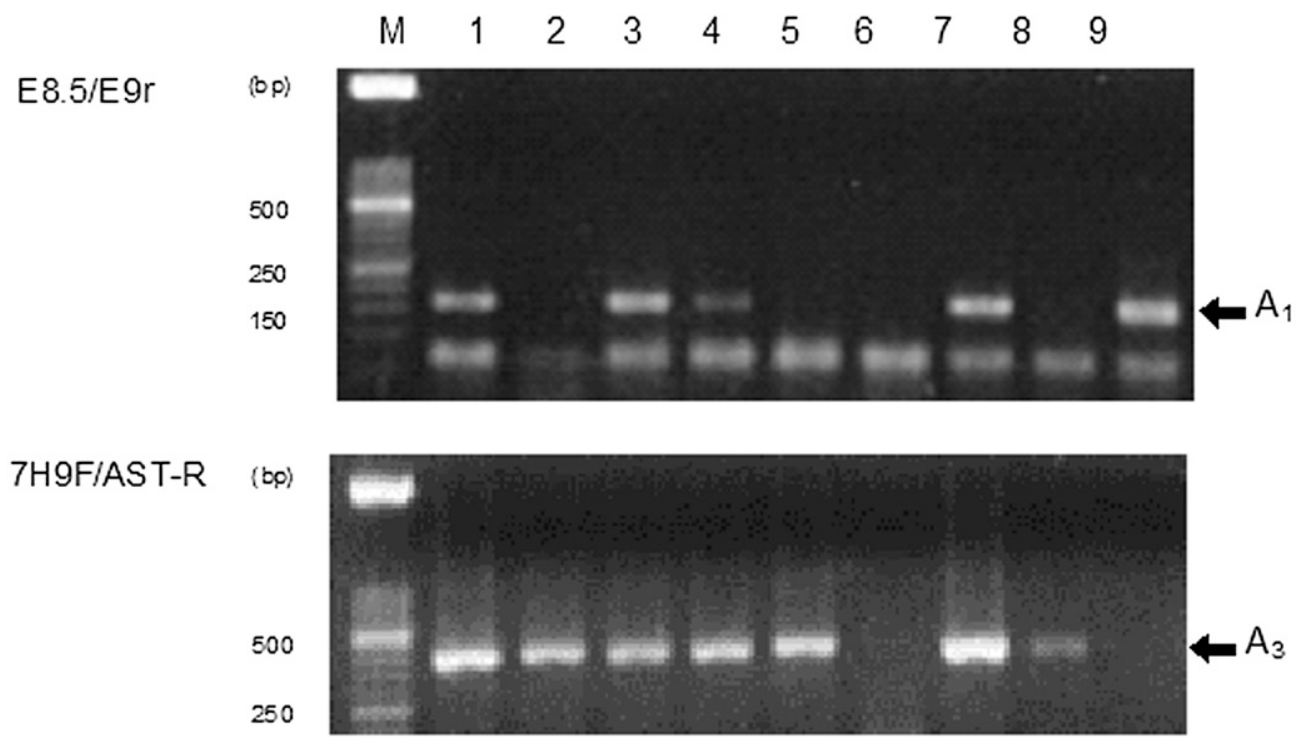

Fig. 2. Segregation of the SCAR markers in the persimmon progeny from 'Taigetsu' $\times$ 'Kanshu.' Lanes 1, 3, 4, 7: offspring presumed to be non-PCNA displaying both the $A_{1}$ and $A_{3}$ markers. Lane 9: offspring presumed to be non-PCNA displaying the $A_{1}$. Lanes 2, 5, 8: offspring presumed to be non-PCNA displaying $A_{3}$. Lanes 6: offspring presumed to be PCNA. M: molecular marker (50 bp ladder; Roche Diagnostics, Tokyo, Japan). SCAR= sequence-characterized amplified region; PCNA = pollination-constant, non-astringent.

segregation ratio of PCNA and non-PCNA offspring in offspring populations.

\section{Materials and Methods}

Plant materials. All of the plant materials were persimmon seedlings derived from a cross between 'Taigetsu' (seed parent) and 'Kanshu' (pollen parent). 'Taigetsu' was selected among the $F_{1}$ offspring populations from a cross of 'Kurokuma' (non-PCNA) and 'Taishu' (PCNA) and released in 2009 (Yamada et al., 2012a). 'Taigetsu' is a non-PCNA cultivar with large fruits, increased productivity, and high eating quality. 'Kanshu' is a PCNA cultivar, which resulted from a cross of 'Shinshu' and ['Fuyu' $\times$ ('Okugosho' $\times$ 'Hanagosho')] (Yamada et al., 2006).

The seedlings were grown in a plastic house, and 1-year-old shoots were top-grafted onto mature 'Fuyu' trees in the persimmon breeding selection field at the NIFTS orchard (Higashihiroshima, Hiroshima, Japan).

The fruit astringency/non-astringency types of the fruit-bearing offspring were identified by a sensory (taste) test and the observations of flesh color. For pollination-variant cultivars, fruits with many seeds are determined to be non-astringent, whereas fruits with only a few seeds were astringent and had many brown specks around the seeds. PCNA flesh is determined to be non-astringent irrespective of the number of seeds with or without brown specks.

PCR for the detection of the SCAR markers. 'Taigetsu' was found to possess two $A S T$-linked regions, $\mathrm{A}_{1}$ and $\mathrm{A}_{3}$, and in our previous report, E4/E9r was used to detect the $\mathrm{A}_{1}$ region, and $7 \mathrm{H} 9 \mathrm{~F} / \mathrm{AST}-\mathrm{R}$ was 
Table 1. Comparison of the $A_{1}$ marker genotype by the E8.5/E9r primers with fruit astringency phenotype of the grafted and fruit-bearing offspring.

\begin{tabular}{lcccc}
\hline A genotype & $\begin{array}{c}\text { Number of grafted } \\
\text { offspring }\end{array}$ & $\begin{array}{c}\text { Number of fruit-bearing } \\
\text { offspring }\end{array}$ & \multicolumn{2}{c}{ Phenotype } \\
\cline { 4 - 5 }+ & 40 & 22 & 0 & PCNA \\
- & 46 & 26 & 11 & 22 \\
\hline PCNA $=$ pollination-PCNA &
\end{tabular}

PCNA $=$ pollination-constant, non-astringent

Table 2. Marker genotypes observed in the offspring population derived from 'Taigetsu' and 'Kanshu' by polymerase chain reaction with the E8.5/E9r and 7H9F/AST-R primers.

\begin{tabular}{|c|c|c|c|c|c|}
\hline \multirow{2}{*}{$\begin{array}{l}\text { Number of } \\
\text { offspring }\end{array}$} & \multicolumn{4}{|c|}{ Marker genotype ${ }^{z}$} & \multirow{2}{*}{$\begin{array}{l}\text { The rate of the expected } \\
\text { PCNA offspring }(\%)\end{array}$} \\
\hline & $\mathrm{A}_{1}+\mathrm{A}_{3}+$ & $\mathrm{A}_{1}+\mathrm{A}_{3}-$ & $\mathrm{A}_{1}-\mathrm{A}_{3}+$ & $\overline{A_{1}-A_{3}-}$ & \\
\hline 522 & 100 & 162 & 179 & 81 & 15.5 \\
\hline
\end{tabular}

${ }^{\mathrm{z}}$ Four genotypes are represented as follows. $A_{1}+A_{3}+$, which exhibit both the $A_{1}$ and $A_{3}$ markers; $A_{1}+A_{3}-$, which exhibit only the $A_{1}$ marker; $A_{1}-A_{3}+$, which exhibit only the $A_{3}$ marker; and $A_{1}-A_{3}-$, which exhibit neither marker.

PCNA $=$ pollination-constant, non-astringent

used to detect the $\mathrm{A}_{3}$ region (Kanzaki et al., 2009, 2010). In the current study, for detecting the $\mathrm{A}_{1}$, we developed a separate E8.5/E9r primer pair (E8.5: 5'-CCAATGGAAGAAG GAATTGGAGAGC-3', E9r: 5'-GCTTAGT CAGCTTAGCCACGCCATTTC-3') based on the sequence between the $\mathrm{E} 8$ and $\mathrm{E} 9$ regions (Kanzaki et al., 2009; see Fig. 1, GenBank Accession no. AB428737). For detecting the $\mathrm{A}_{3}$, the primer pair $7 \mathrm{H} 9 \mathrm{~F} / \mathrm{AstR}$, which was designed to amplify the $A S T$-linked fragment (Kanzaki et al., 2010; Mitani et al., 2014), was used.

Total DNA was extracted from $\approx 1 \mathrm{~cm}^{2}$ of young leaves, sampled from each seedling or grafted plant from offspring plants, using the Nucleon Phytopure plant DNA extraction kit (GE Healthcare UK Ltd., Buckinghamshire, UK). Polymerase chain reaction (PCR) was performed on a total volume of $10 \mu \mathrm{L}$ containing $0.2 \mathrm{~mm}$ of each dNTP, $0.25 \mathrm{U}$ of Ex Taq (Takara Bio, Shiga, Japan), $0.5 \mu \mathrm{M}$ of each primer, $1 \times$ reaction buffer, and $50 \mathrm{ng}$ of the total DNA sample. The PCR conditions were as follows: initial denaturation at $94{ }^{\circ} \mathrm{C}$ for $30 \mathrm{~s}$ followed by 40 cycles of $94{ }^{\circ} \mathrm{C}$ for $20 \mathrm{~s}, 56{ }^{\circ} \mathrm{C}$ for $20 \mathrm{~s}$, and $72{ }^{\circ} \mathrm{C}$ for $20 \mathrm{~s}$ for $\mathrm{E} 8.5 / \mathrm{E} 9 \mathrm{r}$ or $30 \mathrm{~s}$ for $7 \mathrm{H} 9 \mathrm{~F} / \mathrm{AST}-\mathrm{R}$ and a final additional extension at $72{ }^{\circ} \mathrm{C}$ for $5 \mathrm{~min}$. The amplified PCR products were separated on a $2 \%$ agarose gel and visualized by staining with ethidium bromide.

\section{Results and Discussion}

At the beginning of the study, we could detect only the SCAR markers $\mathrm{A}_{1}$ and $\mathrm{A}_{2}$ linked to the dominant $A S T$ alleles in 'Kurokuma', although it has another $A S T$-linked region, $\mathrm{A}_{3}$ (Kanzaki et al., 2009). First, we intended to select non-PCNA offspring derived from the cross between 'Taigetsu' and 'Kanshu', depending on whether the offspring exhibited the $\mathrm{A}_{1}$ marker. A $2.1-\mathrm{kb}$ fragment was generated in the offspring possessing the $\mathrm{A}_{1}$ region using the E4/E9r primer pair (Kanzaki et al., 2009). In this study, the newly developed E8.5/E9r primer pair was used to shorten the reaction time. A fragment of $\approx 160 \mathrm{bp}$ fragment was also amplified in those offspring with E8.5/E9r
(Fig. 2), indicating that the results using the E8.5/E9r primer pair agreed with the results obtained using the E4/E9r primer pair. Consequently, it was concluded that E8.5/E9r could be used for detecting the $A_{1}$ marker.

Forty offspring exhibiting the $A_{1}$ marker $\left(\mathrm{A}_{1}+\right)$ and 46 offspring that did not display the $A_{1}$ marker $\left(A_{1}-\right)$ were grafted onto branches of mature trees. The resulting fruits were subjected to the sensory test to confirm the astringency trait. Four years after grafting, 22 of the $\mathrm{A}_{1}+$ offspring bore fruits, all of which were found to have the non-PCNA phenotype (Table 1). In contrast, among the resulting 26 fruit-bearing $A_{1}$ - offspring, 11 and 15 were found to have the PCNA and non-PCNA phenotype, respectively. The segregation ratio (11:15) was not significantly different from $1: 1\left(P=0.433\right.$ by $\chi^{2}$ test $)$. All of the 15 non-PCNA offspring were later confirmed by the 7H9F/AST-R primers to possess the $\mathrm{A}_{3}$ marker. The results demonstrated good agreement between the discrimination by SCAR markers and the fruit astringency trait. From these results in addition to the previous study (Mitani et al., 2014), it was found that the detection of the $\mathrm{A}_{1}, \mathrm{~A}_{2}$, and $\mathrm{A}_{3}$ markers among offspring derived from the backcross of non-PCNA $\mathrm{F}_{1}$ offspring to PCNA with three primer pairs could be accurate, although the genotype of the non-PCNA cross parents should be elucidated in advance.

A serious problem for breeding new PCNA cultivars is inbreeding depression caused by narrow genetic pools among 2005). So the backcross of non-PCNA $F_{1}$ offspring derived from PCNA $\times$ non-PCNA to PCNA should be adopted for PCNA breeding. However, even in the case of the crosses among PCNA cultivars/selections, it takes more than 10 years from the cross to the release of a new cultivar and would take longer in the case of the backcrosses. Thus, the development of markers that can discriminate PCNA from non-PCNA is indispensable.

Subsequently, the applicability of using the E8.5/E9r and 7H9F/AST-R primer pairs for the selection of PCNA offspring in the Japanese PCNA cultivars (Yamada, 1993, breeding program was evaluated. Among 522 offspring, 100 offspring exhibited both the $A_{1}$ and $A_{3}$ markers (Table 2). Furthermore, 162 offspring displayed only the $A_{1}$ marker, and 179 showed only the $\mathrm{A}_{3}$ marker. Eighty-one offspring did not display either of the markers. The percentage of offspring that were presumed to be the PCNA genotype was $15.5 \%$ ( 81 of 522 ), which was not significantly different from $18.3 \%$ (46 of 251) observed for 'Taiten' $\times$ 'Kanshu' (Mitani et al., 2014) $(P=0.377)$.

The autohexaploid model for inheritance of the AST locus was generally fitted to the expected ration with some exceptions using marker genotyping (Akagi et al., 2009). The theoretical offspring segregation ratio from $\mathrm{A}_{1} \mathrm{~A}_{3}$ aaaa $\times$ aaaaa is $20 \% \quad \mathrm{~A}_{1} \mathrm{~A}_{3}$ aaaa, $30 \%$ $\mathrm{A}_{1}$ aaaaa, $30 \% \mathrm{~A}_{3}$ aaaaa, and $20 \%$ aaaaa in random chromosome assortment for autohexaploid, which approached but was significantly different from the observed data in this study $(P=0.032)$. The observed percentage of nulliplex expressing PCNA was $15.5 \%$ (Table 2), which was slightly lower than $20 \%$, but the percentage of PCNA offspring was $22.9 \%$ (11 of 48) for phenotypic segregation of the astringency type (Table 1$)$, which was not significantly different from $20 \%\left(P=0.613\right.$ by $\chi^{2}$ test). Sato et al. (2013) reported that PCNA offspring from the cross between 'Taigetsu' or 'Taiten' with 'Kanshu' had significantly small fruit weight and were likely to cause fruit cracking at the fruit apex, and it is possible that some unknown factors modify the segregation ratio. However, $\approx 20 \%$ PCNA offspring were obtained from the cross of 'Taigetsu' and 'Kanshu', and it was concluded that those offspring were discriminated effectively by the molecular markers.

In conclusion, a practical application of marker-assisted selection in the PCNA persimmon-breeding program at NIFTS has been shown. PCNA offspring can be selected by the two PCR primers E8.5/E9r and $7 \mathrm{H} 9 \mathrm{~F} / \mathrm{AST}-\mathrm{R}$ in the progeny derived from 'Taigetsu' $\times$ 'Kanshu.' With the marker-assisted selection, only scions of PCNA offspring selected from the backcross can be grafted onto mature trees to promote early fruiting in a selection field. Assuming that the backcross of the non-PCNA $\mathrm{F}_{1}$ offspring derived from PCNA $\times$ non-PCNA to PCNA yielded $20 \%$ PCNA offspring, the selection efficiency could be increased $\approx 5$-fold and the cost of maintaining fields and trees reduced. It is no doubt that raising a large number of offspring continuously in the field and evaluations using actual trees and fruits are necessary. The marker-assisted selection described in this article will help persimmon breeders to select only PCNA offspring and to improve the efficiency of PCNA persimmon breeding.

\section{Literature Cited}

Akagi, T., S. Kanzaki, M. Gao, R. Tao, D.E. Parfitt, and K. Yonemori. 2009. Quantitative real-time PCR to determine allele number for the astringency locus by analysis of a linked marker in Diospyros kaki Thunb. Tree Genet. Genomes 5:483-492. 
Akagi, T., Y. Takeda, K. Yonemori, A. Ikegami, A Kono, M. Yamada, and S. Kanzaki. 2010. Quantitative genotyping for the astringency locus in hexaploid persimmon cultivars using quantitative real-time PCR. J. Amer. Soc. Hort. Sci. 135:59-66.

Akagi, T., T. Tao, T. Tsujimoto, A. Kono, and K. Yonemori. 2012. Fine genotyping of a highly polymorphic ASTRINGENCY-linked locus reveals variable hexasomic inheritance in persimmon (Diospyros kaki Thunb.) cultivars. Tree Genet. Genomes 8:195-204.

Hume, H.H. 1914. A Kaki classification. J. Hered. 5:400-406.

Ikeda, I., M. Yamada, A. Kurihara, and T. Nishida. 1985. Inheritance of astringency in Japanese persimmon. J. Jpn. Soc. Hort. Sci. 54:39-45 [in Japanese with English abstract].

Kajiura, M. 1946. Persimmon cultivars and their improvement (2). Breed. Hort. 1:175-182 [in Japanese].

Kanzaki, S., T. Akagi, T. Masuko, M. Kimura, M. Yamada, A. Sato, N. Mitani, N. Ustunomiya, and K. Yonemori. 2010. SCAR markers for practical application of marker-assisted selection in persimmon (Diospyros kaki Thunb.) breeding. J. Jpn. Soc. Hort. Sci. 79:150-155.

Kanzaki, S., A. Sato, M. Yamada, N. Utsunomiya, A. Kitajima, A. Ikegami, and K. Yonemori. 2008. RFLP markers for the selection of pollination-constant and non-astringent (PCNA)type persimmon and examination of the inheritance mode of the markers. J. Jpn. Soc. Hort. Sci. 77:28-32.

Kanzaki, S., M. Yamada, A. Sato, N. Mitani, N. Utsunomiya, and K. Yonemori. 2009. Conversion of RFLP markers for the selection of pollination-constant and non-astringent type persimmons (Diospyros kaki Thunb.) into PCRbased markers. J. Jpn. Soc. Hort. Sci. 78:68-73.

Kanzaki, S., K. Yonemori, A. Sugiura, A. Sato, and M. Yamada. 2001. Identification of molecular markers linked to the natural astringency-loss of Japanese persimmon (Diospyros kaki) fruit. J. Amer. Soc. Hort. Sci. 126:51-55.

Kitagawa, H. and P.G. Glucina. 1984. Persimmon culture in New Zealand. Science Information Publishing Centre, Wellington, New Zealand.

Mitani, N., A. Kono, M. Yamada, A. Sato, S. Kobayashi, Y. Ban, T. Ueno, M. Shiraishi, S. Kanzaki, T. Tsujimoto, and K. Yonemori. 2014. Practical marker-assisted selection using two SCAR markers for fruit astringency type in crosses of 'Taiten' $\times$ PCNA cultivars in persimmon breeding. Sci. Hort. 170:219-223.

Sato, A., A. Kono, N. Mitani, Y. Ban, and M. Yamada. 2013. Comparison of fruit traits between pollination constant non-astringent (PCNA) and non-PCNA offspring derived from two backcrosses. Acta Hort. 996:123-126.

Tamura, M., R. Tao, K. Yonemori, N. Utsunomiya, and A. Sugiura. 1998. Ploidy level and genome size of several Diosphyros species. J. Jpn. Soc. Hort. Sci. 67:306-312.
Yamada, M. 1993. Persimmon breeding in Japan. Jpn. Agr. Res. Q. 27:33-37.

Yamada, M. 2005. Persimmon genetic resources and breeding in Japan. Acta Hort. 685:51-64.

Yamada, M. and A. Sato. 2002. Segregation for fruit astringency type in progenies derived from crosses of 'Nishimurawase' $\times$ pollination constant non-astringent genotypes in oriental persimmon (Diospyros kaki Thunb.). Sci. Hort. 92:107-111.

Yamada, M., A. Sato, H. Yamane, N. Mitani, H. Iwanami, M. Shiraishi, N. Hirakawa, T. Ueno, A. Kono, M. Yoshioka, and I. Nakajima. 2012a. New Japanese persimmon cultivar, 'Taigetsu'. Bull. NARO Inst. Fruit Tree Sci. 14:25-38 [in Japanese with English abstract].

Yamada, M., A. Sato, H. Yamane, N. Mitani, H. Iwanami, M. Shiraishi, N. Hirakawa, T. Ueno, A. Kono, M. Yoshioka, and I. Nakajima. 2012b. New Japanese persimmon cultivar, 'Taiten.'. Bull. NARO Inst. Fruit Tree Sci. 14:39-52 [in Japanese with English abstract].

Yamada, M., A. Sato, H. Yamane, K. Yoshinaga, N. Hirakawa, H. Iwanami, T. Ozawa, M. Kakutani, N. Mitani, M. Yoshioka, and I. Nakajima. 2006. New Japanese persimmon cultivar, 'Kanshu'. Bull. National Institute of Fruit Tree Science 5:95-106 [in Japanese with English abstract].

Yonemori, K., A. Sugiura, and M. Yamada. 2000. Persimmon genetics and breeding, p. 191-225. In: Janick, J. (ed.). Plant breeding reviews 19. John Wiley \& Sons, Inc., New York, NY. 